\title{
Natural Frequencies of Rectangular Laminated Plates-Introduction to Optimal Design in Aeroelastic Problems
}

\author{
Aleksander Muc \\ Institute of Machine Design, Cracow University of Technology, 31-155 Kraków, Poland; \\ olekmuc@mech.pk.edu.pl; Tel.: +48-126283360
}

Received: 7 August 2018; Accepted: 2 September 2018; Published: 10 September 2018

\begin{abstract}
Free vibration (or eigenvalue analysis) is a prerequisite for aeroelastic analysis. For divergence analysis, slope influence coefficients (rotation at point $i$ due to unit load at point $j$ ) are calculated using free vibration mode shapes and corresponding frequencies. The lowest eigenvalue is of interest and gives the divergence speed. The present paper considers the maximization problem of eigenfrequencies for composite panels. The influence of boundary conditions and constant or variable stiffnesses on optimization results are investigated herein. A new convenient set of design variables is employed in the analysis. The computations are carried out with the use of the Rayleigh-Ritz method and Finite Element analysis (2D quadrilateral and 3D solid elements).
\end{abstract}

Keywords: free vibrations; laminated plates; optimization

\section{Introduction}

The most widespread use of composites continues to be for aircraft structural components for both the airframe and the engine components. Aeroelasticity is a crucial discipline in the design and maintenance of an aircraft. Knowledge in this subject might have prevented incidents. Most aircraft and space vehicle structures, where composites have tremendous applications, are subjected to severe aerodynamic loads during their flight. These aerodynamic forces influence the static and dynamic response of the composite structures and panel flutter is one of those effects. Plate flutter is a self-excited oscillation of an elastic structure which results from the coupling effects of aerodynamic, elastic, and inertial forces. It is an aeroelastic phenomenon that usually causes fatigue damage, not instantaneous fracture of flight vehicles.

In panel flutter analysis, major concerns are the effects of physical materials (like isotropic, orthotropic, and anisotropic), geometrical model (like the shape and dimensions of the panel), and boundary conditions (like simple support, clamp, free, and guide) on flutter. Except for these classical parameters, there are more factors and complex physical environments considered in recent decades, like aerodynamic uncertainties, new composite materials, fluid-thermal-structural coupling, boundary layer, and shear deformation.

For composite panels, in order solve more common aeroelastic problems (divergence, flutter, and aeroelastic response at subsonic and supersonic speeds), the analysis actually starts with finite element modeling and free vibration studies. Then, eigenmode shapes are interpolated from the structural grid points to aerodynamic control points to formulate modal equations of motion and to investigate critical divergence velocities, flutter speed, flutter frequency, etc. When the aerodynamic damping is negligible, the panel flutter analysis is normally made using the frequency coalescence method. For zero dynamic pressure parameter, the eigenvalues of the system are real and positive. They are basically the natural frequencies of the system in vacuum. As the dynamic pressure parameter 
is increased from zero, the frequencies keep changing: some decrease in value and some increase in value. For some value of dynamic pressure parameter, known as the critical dynamic pressure parameter, two of the eigenvalues come very close to each other and become complex conjugates. The merging of the frequencies is known as frequency coalescence.

The mode shape is of high importance, not to say the most important parameter. Long and slim structures exposed to high aerodynamic loads are sensitive to flutter, typically the first stages of compressors and last stages of turbines. According to the nature of the structure itself, the mode shape may be of different levels of complexity. In external aerodynamics such as airplane wings, mode shape can be a combination of the first eigenmodes of the structure (the involved modes must lie in frequency such that they can be coupled by the flow); whereas in internal flows, the mode shapes are typically pure eigenmodes. The main difference between internal and external aeroelasticity is the material stiffness and is expressed through the so-called mass ratio defining the ratio between the mass of the structure and the mass of surrounding air inside a circle with radius half the chord. In turbo machines, the blades are stiff and the mass ratio is consequently larger compared with that for airplane wings. In 1994, Lovejoy [1] constructed the atlas of mode shapes for generally laminated, thick, rectangular, skew, and trapezoidal plates. The problems connected with finite element modeling of skew composite plates were discussed by Fantuzzi et al. [2].

Vibrations of composite multilayered plates have been of great interest of numerous authors and most of their work has been referenced, e.g., by Yu [3], Kapania and Raciti [4], and Reddy and Khdeir [5]. The analysis of natural vibrations of doubly curved composite and sandwich structures was conducted by Tornabene et al. [6,7].

Optimization of natural frequencies is also a central concept in the design of plated composite structures. Design parameters (denoted by the vector s) such as layer thicknesses and ply angles can be employed to achieve an optimized structure.

Structures with some frequency requirements can be optimized using three computationally different strategies—see Grandhi [8], Szyszkowski and King [9]. To avoid vibrational resonance in aerospace and naval structures, laminated plates are usually designed for maximum fundamental frequency constraints $\omega$, i.e.,

$$
\underset{\mathbf{s}}{\operatorname{Max}} \omega(\mathbf{s})
$$

Bert [10,11], Reiss and Ramachandran [12], and Grenestedt [13] maximized the fundamental frequency of thin symmetric laminates by treating the fiber orientation angle of each layer as a continuous design variable. In the cited works [10-13], frequency analysis was performed using a closed-form formula for thin rectangular laminated plates based on the classical lamination theory.

The frequency tuning is the second optimization problem [9]. It can be treated as the alternative or sometimes equivalent optimization problem to the above:

$$
\underset{\mathbf{s}}{\operatorname{Max}}\left[\omega_{l}(\mathbf{s})-\omega_{l-1}(\mathbf{s})\right], l=2,3, \ldots
$$

To increase the flutter speed, designers strive to increase the difference between modal frequencies by increasing the stiffness and changing the mass distribution (Wright and Cooper [14]). Flutter can also be prevented by eliminating some modes of vibration that cause the phenomenon, through the manipulation of certain design parameters (Chowdary et al. [15]) or through the use of control surfaces and actuators (Foster and Yang [16]).

As an attempt to improve future designs and prevent flutter, the literature is rich in papers on the flutter analysis of beams (Moosavi et al. [17]), plates (Chowdary et al. [15]; Zhao and Cao [18]), and wings (Forster and Yang [16]; Alyanak and Pendleton [19]) of different materials and subjected to different loading conditions. Chowdary et al. [15] and Singha and Ganapathi [20] studied the flutter of composite plates. Both studies pointed out a relation between the skew angle, boundary conditions, fiber angle, and the flutter behavior of composite panels. 
Hyer and Charette [21] introduced the concept of variable-stiffness panels. This was then generalized by Abdalla et al. [22]. However, in the latter approach [22], the variable stiffness was parameterized by the lamination parameters and was not attributed to fiber orientations and ply thicknesses—see the definition in Equation (15). Kameyama and Fukunaga [23] optimized the plate wing structure using lamination parameters but the thickness variations of the composite plate were represented by the parabolic function.

In composite laminates, piezoelectric elements usually consist of patches symmetrically bonded to the top and bottom surfaces (Figure 1). The voltages applied to the piezoelectric elements may induce inplane, bending, and localized shear deformations in the laminate [24]. For symmetric laminates, applying the same in-phase voltage for both patches will cause only inplane deformation, whereas equal out-of-phase voltage will cause pure bending. Combined inplane and bending deformation may be induced when different voltages are applied to each patch or when one of the elements is used as actuator and the other as sensor. The same situation occurs if a single piezoelectric actuator is mounted on a surface. The presence of inplane deformation may have a significant influence on the mechanical behavior of plates, affecting the flexural stiffness and, hence, the dynamic and stability characteristics of isotropic [25] and laminated plates [26]. A great deal of research has been performed to demonstrate the active control of lightweight smart structures-see, e.g., [27-32]. In the cited references, optimum fields of residual inplane stresses that maximize the first natural frequency and buckling load of plates and shells were determined.

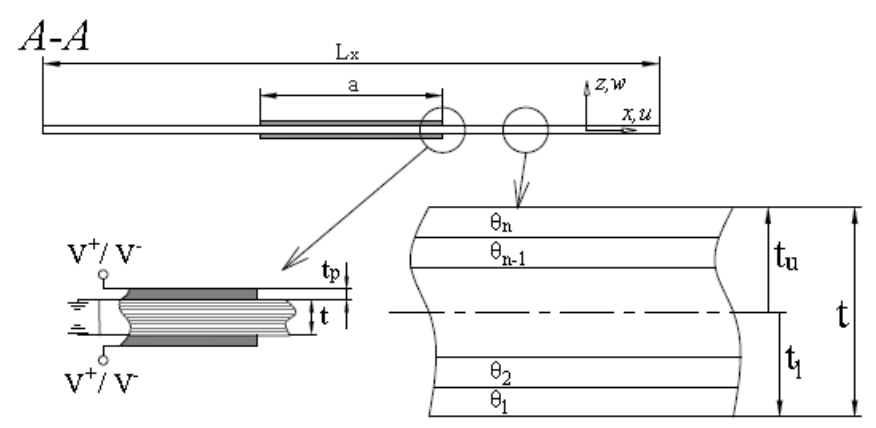

Figure 1. Composite structure reinforced by piezoelectric (PZT) layers.

In many aerospace and mechanical applications, the structures need to be stiff at one location and flexible at another location depending on the position where large displacements and flapping motions are experienced. Further, as the tapered composite structures are formulated by dropping-off plies at discrete locations and adding resins appropriately, the stiffness of the structure could be altered (Figure 2). Hence, the designer is able to achieve better strength with minimal weight, which could not be attainable in uniform composite structures. As the tapered composite structures yield variable stiffness along longitudinal and transverse directions, investigations into the various properties of laminated composite tapered structures would be beneficial for the designer to get the variable stiffness and damping properties and attenuate the vibration efficiently—see, e.g., Refs. [33-38].

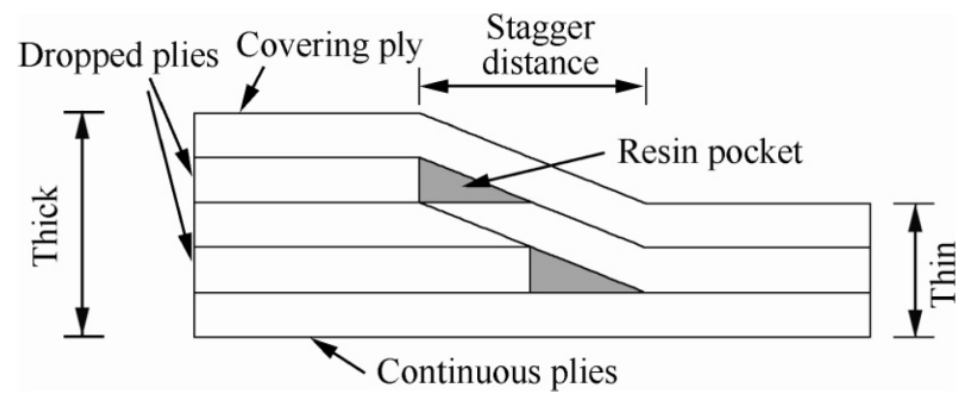

Figure 2. Tapered composite structure. 
Weight optimization of structures with frequency constraints is considered to be a challenging problem. Mass reduction conflicts with the frequency constraints, especially when they are lower bounded—see, e.g., Muc [39].

\section{Governing Relations}

In order to develop the governing equations for this investigation, several assumptions are made as follows:

- The plate is constructed of flat, uniform-thickness layers of orthotropic sheets bonded together. The direction of principal stiffness of the individual layers does not in general coincide with the plate edges (see Figure 3). The plate is thin; i.e., the thickness $t$ is much smaller than the other physical dimensions $L_{x}$ and $L_{y}$.

- In the theoretical considerations, the Kirchhoff hypothesis is used, i.e., transverse shear and normal strains are negligible; however, in the finite element analysis, the first-order transverse shear deformation theory is used.

- The thicknesses of individual layers are identical and equal to $t / N$, where $N$ denotes the total number of plies in the laminate; the distances in the individual laminas are measured from the geometrical mid-plane of the plate.
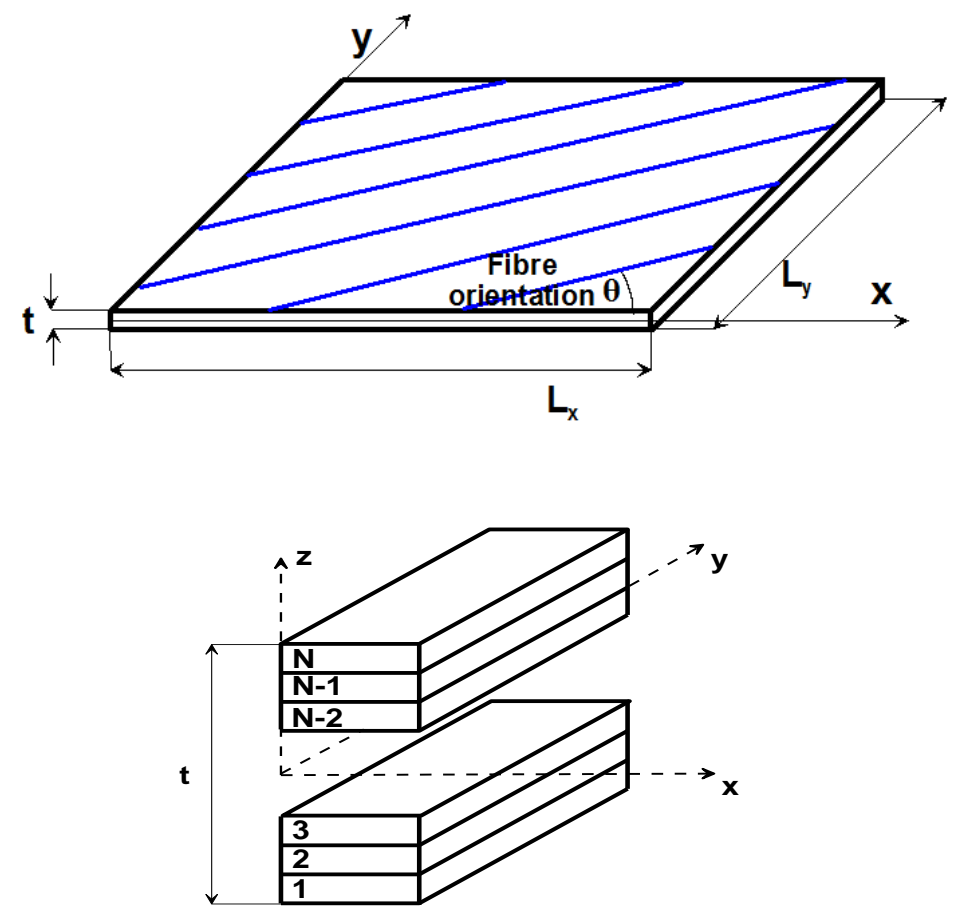

Figure 3. Panel geometry and the stacking sequence.

The fundamental relations (understood in the sense of the resulting frequency equations) are derived from Hamilton's principle:

$$
\int_{\tau_{1}}^{\tau_{2}} \delta(T-U) d \tau=0
$$

where the kinetic energy $T$ can be written as

$$
T=\frac{1}{2} \int_{\Omega}\left(\frac{\partial \bar{w}}{\partial \tau}\right)^{2} d \Omega
$$


and the total strain energy $U$

$$
U=\frac{1}{2} \int_{\Omega}\left[\varepsilon_{m} A \varepsilon_{m}^{T}+\varepsilon_{m} B \kappa^{T}+\kappa B \varepsilon_{m}^{T}+\kappa D \kappa^{T}\right] d \Omega
$$

where $\varepsilon_{m}, \kappa$ are the membrane linear strain vector and the curvature changes vector, respectively. These components can be written by the displacement components $\bar{u}, \bar{v}, \bar{w}$ in the Cartesian coordinate system $x, y$, and $z$ as

$$
\left\{\varepsilon_{m}\right\}=\left\{\frac{\partial \bar{u}}{\partial x}, \frac{\partial \bar{v}}{\partial y}, \frac{\partial \bar{u}}{\partial y}+\frac{\partial \bar{v}}{\partial x}\right\},\{\kappa\}=-\left\{\frac{\partial^{2} \bar{w}}{\partial x^{2}}, \frac{\partial^{2} \bar{w}}{\partial y^{2}}, 2 \frac{\partial^{2} \bar{w}}{\partial x \partial y}\right\}
$$

where $\tau$ denotes the physical time and $\Omega$ is the $2 \mathrm{D}$ occupied by the mid-plane of the plate.

$\mathbf{A}, \mathbf{B}$, and $\mathbf{D}$ are the extensional, flexural-extensional coupling, and flexural stiffness matrices, which are defined in the classical way, i.e.,

$$
\begin{gathered}
\mathbf{A}=\sum_{k=1}^{N} Q_{i j}^{(k)}\left(z_{k}-z_{k-1}\right), i, j=1,2,6 \\
\mathbf{B}=\frac{1}{2} \sum_{k=1}^{N} Q_{i j}^{(k)}\left(z_{k}^{2}-z_{k-1}^{2}\right), i, j=1,2,6 \\
\mathbf{D}=\frac{1}{3} \sum_{k=1}^{N} Q_{i j}^{(k)}\left(z_{k}^{3}-z_{k-1}^{3}\right), i, j=1,2,6
\end{gathered}
$$

Assuming that all layers in the laminate are made of identical composite materials, the terms $Q_{i j}^{(k)}$ can be expressed as follows:

$$
\begin{gathered}
Q_{11}^{(k)}=U_{1}+U_{2} \cos 2 \theta^{(k)}+U_{3} \cos 4 \theta^{(k)}, Q_{12}^{(k)}=U_{4}-U_{3} \cos 4 \theta^{(k)}, \\
Q_{22}^{(k)}=U_{1}-U_{2} \cos 2 \theta^{(k)}+U_{3} \cos 4 \theta^{(k)}, Q_{66}^{(k)}=U_{5}-U_{3} \cos 4 \theta^{(k)}, \\
Q_{16}^{(k)}=\frac{1}{2} U_{2} \sin 2 \theta^{(k)}+U_{3} \sin 4 \theta^{(k)}, Q_{26}^{(k)}=\frac{1}{2} U_{2} \sin 2 \theta^{(k)}-U_{3} \sin 4 \theta^{(k)} \\
U_{1}=\frac{1}{8}\left(3 Q_{11}+3 Q_{22}+2 Q_{12}+4 Q_{66}\right), U_{2}=\frac{1}{2}\left(Q_{11}-Q_{22}\right), U_{3}=\frac{1}{8}\left(Q_{11}+Q_{22}-2 Q_{12}-4 Q_{66}\right), \\
U_{4}=\frac{1}{8}\left(Q_{11}+Q_{22}+6 Q_{12}-4 Q_{66}\right), U_{5}=\frac{1}{2}\left(U_{1}-U_{4}\right) \\
Q_{11}=\frac{E_{1}}{1-v_{12} v_{21}}, Q_{12}=\frac{v_{12} E_{2}}{1-v_{12} v_{21}}, Q_{22}=\frac{E_{2}}{1-v_{12} v_{21}}, Q_{66}=G_{12}
\end{gathered}
$$

where $\theta^{(k)}$ denotes the fiber orientation of the $k$ th ply.

When the plate is oscillating in a normal mode, the motion is harmonic, so the solutions may be taken in a form of a harmonic function of time $\tau$ with a natural circular frequency $\omega_{m n}(m, n$ denote the modes of vibration), such as

$$
\{\bar{u}, \bar{v}, \bar{w}\}(x, y, \tau)=\{u, v, w\}(x, y) \cos \left(\omega_{m n} \tau\right) .
$$

Available formulations of the governing equations and boundary conditions are applicable for general laminated plates. However, most of the solutions have been obtained for special classes of plates which result in considerable simplifications to the governing equations.

Due to the coupling between moment and stress resultants, two differential equations are required to describe the behavior of the plate under various boundary and load conditions. The first equation will be developed using plate equations of motion. The second relation is derived from the compatibility conditions where the stress resultants are replaced by the Airy function $F$. A detailed discussion of those problems is presented, e.g., by Muc et al. [31]. Finally, the Hamilton functional (Equation (3)) is a function of two variables (unknowns): $w$ and $F$. In the governing relations, all 
components of the matrices $\mathbf{A}, \mathbf{B}$, and $\mathbf{D}$ exist and are not equal to zero. Since certain special classes of plates have been studied almost exclusively in the literature, the simplifications to the general solutions due to the special constructions will be discussed. The special classes of plates considered are symmetric laminated plates and angle-ply plates.

For symmetrically laminated plates, all terms of the $\mathbf{B}$ matrix are equal to zero. Therefore, plate equations of motion and the compatibility equation are not coupled. Although the motion equation is considerably simpler than for general laminated plates, it differs from the governing equation of an orthotropic plate by the additional $D_{16}$ and $D_{26}$ terms, i.e.,

$$
D_{16} \neq 0, D_{26} \neq 0 \text {. }
$$

Angle-ply plates by definition are constructed so as to have an even number of layers of the same thickness and elastic properties, and with the orthotropic axis of symmetry in each ply alternately oriented at $+\theta$ and $-\theta$. For angle-ply plates, this stacking sequence results in some of the coefficients being zero as follows:

$$
\begin{gathered}
A_{16}=A_{26}=D_{16}=D_{26}=0 \\
B_{11}=B_{12}=B_{22}=B_{66}=0
\end{gathered}
$$

whereas

$$
B_{16} \neq 0, B_{26} \neq 0 \text {. }
$$

Thus, two governing equations are coupled and must be solved simultaneously. As it is proved by Whitney and Leissa [40], the coupling effects (Equation (12)) depend on the number of plies $N$. In further considerations, it is assumed that $N \geq 12$ and those effects are neglected.

\section{Method of the Solution}

For symmetric laminates, the resulting frequency equations can be derived from the Hamilton principle (Equation (3)) with the use of the relations given in Equations (4), (5), and (9):

$$
\omega^{2}=\frac{\int_{\Omega} \kappa D \kappa^{T} d \Omega}{\int_{\Omega} w^{2} d \Omega} .
$$

For angle-ply laminates and $N \geq 12$, Equation (13) is simplified by inserting $D_{16}=D_{26}=0$ (see Equation (11)).

The response of the plates due to the existence of nonzero bending-twisting terms $D_{16}, D_{26}$ was analyzed by Grenestedt [41]. The author compared the perturbations (see Equation (8))

$$
\frac{D_{16}}{D_{0}}, \frac{D_{26}}{D_{0}}, D_{0}=D_{11}+D_{12}+D_{22}+D_{66}=2 U_{1}+U_{4}+U_{5}
$$

and proved that nonzero $D_{16}, D_{26}$ stiffnesses never make a laminate perform better, so we, from now on, let them be zero.

For two laminate configurations (symmetric or angle-ply laminates), the standard methods of solution can be used: (1) the Rayleigh-Ritz method—the variational method (e.g., the symbolic package Mathematica), (2) the Bubnov-Galerkin method, or (3) Finite Element Analysis. The applicability and the simplicity of the analysis depend directly on the type of boundary conditions and on the form of the approximation functions. J. Li and Y. Narita [42] proposed a general form of approximation functions for different boundary conditions.

It is assumed that the edges $1,2,3$, and 4 denote the boundaries along $y=0, x=L_{x}, y=L_{y}$, and $x=0$, respectively (Figure 3). The symbols F, C, and S mean that the edge is free, clamped, or simply supported, respectively. 


\section{Optimal Design}

\subsection{Definition of Design Variables}

Each layer of a laminate can be identified by its location in the laminate, its fiber orientation, and its material properties. It is possible to determine the optimum orientation sequence for any given load condition. The effectiveness of the optimal design, especially for composite structures, is strongly dependent on the proper choice of two elements: (i) the definition of design variables and (ii) the application of the appropriate optimization algorithm. The simplest definition of design variables depends on the direct application of the real, continuous variables (i.e., fiber orientations $\theta_{l}$ and thicknesses $t_{l}$ in the $l$ th ply, $\left.l=1,2, \ldots, N\right)$. Now, such an approach is commonly used in finite element codes, such as, e.g., ANSYS, ABAQUS, etc. This method is not very convenient for many engineering or analytical applications and is replaced by the introduction of so-called lamination parameters (Miki [43], Vanderplaats and Fukunaga [44]), i.e.,

$$
\begin{gathered}
\xi_{[1,2,3,4]}^{\{A, D\}}=\int_{-t / 2}^{t / 2}[\cos 2 \theta(z), \cos 4 \theta(z), \sin 2 \theta(z), \sin 4 \theta(z)]\left\{\frac{1}{t}, \frac{4 z}{t^{2}}, \frac{12 z^{2}}{t^{3}}\right\} d z \\
\theta(z)=\left\{\begin{array}{ccc}
\theta_{i} & \text { where } & z \in\left[z_{i-1}, z_{i}\right] \\
0 & \text { where } & z \notin\left[z_{i-1}, z_{i}\right]
\end{array}\right.
\end{gathered}
$$

The lamination parameters are usually determined for thin-walled composite structures (i.e., beams, plates, or shells) with the use of the Love-Kirchhoff kinematical hypothesis. For an arbitrary laminate, the structural stiffness is characterized by 12 independent parameters instead of $2 N$ variables for the previous real, continuous variables $\left(\theta_{l}\right.$ and $\left.t_{l}\right)$. In fact, the analytical studies deal mainly with the use of four of them, i.e., $\xi_{[1]}^{\{A\}}, \xi_{[2]}^{\{A\}}, \xi_{[1]}^{\{D\}}$, and $\xi_{[2]}^{\{D\}}$ corresponding to laminates where the stiffnesses $B_{i j}, A_{16}, A_{26}, D_{16}$, and $D_{26}$ are assumed to be equal to zero. Let us note that for the symmetric, balanced laminate (which satisfies the previous assumptions) having six layers, (six independent, unknown variables, i.e., $t_{1}, t_{2}, t_{3}$ and $\left.\theta_{1}, \theta_{2}, \theta_{3}\right)$ are expressed by four lamination parameters. If bending problems are analyzed (e.g., free vibrations or buckling of compressed rectangular plates), all six unknowns are described by two lamination parameters. Of course, in such a case it is impossible to find the exact values of $t_{1}, t_{2}, t_{3}$ and $\theta_{1}, \theta_{2}, \theta_{3}$. It is worth noticing herein that knowing two optimal laminate parameters (i.e., $\xi_{[1]}^{\{D\}}, \xi_{[2]}^{\{D\}}$ ), it is impossible to derive the membrane lamination parameters $\xi_{[1]}^{\{A\}}$ and $\xi_{[2]}^{\{A\}}$. They may be only found if, from the known lamination parameters $\xi_{[1]}^{\{D\}}$ and $\xi_{[2]}^{\{D\}}$, it is possible to guess simply the laminate configuration in the sense of thicknesses $t_{1}, t_{2}, t_{3}$ and fiber orientations $\theta_{1}$, $\theta_{2}, \theta_{3}$ in each individual ply. A lot of effort has been directed toward the derivation of feasible regions for 12 lamination parameters, especially by Diaconu et al. [45,46] and Bloomfield et al. [47]; however, they seem to be impractical since the optimal solutions are mainly controlled by vibration constraints, and not by geometrical relations between sets of lamination parameters.

Therefore, we propose to use the graphical optimization method to solve the optimization problem that can be formulated in the following way: to maximize the natural frequencies, expressed by Equation (1), with respect to $N$ design variables characterizing fiber orientations in each of the $N$ plies constituting the laminate. It is assumed that the thickness of each ply is identical (i.e., equal to $t / N)$, as well as their mechanical properties. In such a situation, Equation (7c) is reduced to the following form:

$$
D_{i j}=\frac{t^{3}}{12} \sum_{k=1}^{N} Q_{i j}^{(k)}, i, j=1,2,6 .
$$

For angle-ply laminate configuration and $N \geq 12$, Equation (13) is a function of one design variable, $\cos (2 \theta)$ only-see Equations (8) and (11), and use the classical trigonometric reduction formulas. It is obvious that searching for the maximum/minimum of Equation (13) is not a problem and can be done easily both analytically and numerically. 
In the second case of the symmetrically laminated plates, let us introduce two design variables, $x$ and $y$, in the following way:

$$
x=\frac{4}{t^{3}} \sum_{l=1}^{N}\left(z_{l}^{3}-z_{l-1}^{3}\right) \cos \left(2 \theta^{(l)}\right), y=\frac{4}{t^{3}} \sum_{l=1}^{N}\left(z_{l}^{3}-z_{l-1}^{3}\right) \cos ^{2}\left(2 \theta^{(l)}\right),
$$

Again assuming identical thicknesses and mechanical properties of the plies, Equation (7c) can be expressed as follows:

$$
\begin{aligned}
& D_{11}=\frac{t^{3}}{12}\left(U_{1}-U_{3}+U_{2} x+2 U_{3} y\right), D_{12}=\frac{t^{3}}{12}\left(U_{4}+U_{3}-2 U_{3} y\right), \\
& D_{22}=\frac{t^{3}}{12}\left(U_{1}-U_{3}-U_{2} x+2 U_{3} y\right), D_{66}=\frac{t^{3}}{12}\left(U_{5}+U_{3}-2 U_{3} y\right)
\end{aligned}
$$

Now, it is well known that the design space is represented by the interior and the boundary of a parabola (Figure 4). The axes of the coordinate system are defined by Equation (17). Each point of the interior of the parabola is characterized by two real numbers representing uniquely the laminate stacking sequence. The boundary of the parabola described by the function $y=x^{2}$ corresponds to the previous case, i.e., the angle-ply symmetric fiber orientations $\pm \theta$. The diagram in Figure 4 can also be used for designing laminates with predetermined ply orientation angles (i.e., having discrete ply orientations). The feasible region for laminates with fixed ply angles is a polygon with vertices located on the envelope (the parabola). The possible forms of the polygons are drawn in Figure 4. For laminates with pairs of $0^{\circ}, 90^{\circ}$ plies, and $\pm 45^{\circ}$, the design space is a triangle. For laminates with $0^{\circ}, \pm 15^{\circ}, \pm 30^{\circ}, \pm 45^{\circ}, \pm 60^{\circ}, \pm 75^{\circ}$, and $90^{\circ}$ plies, the space forms a polygon.

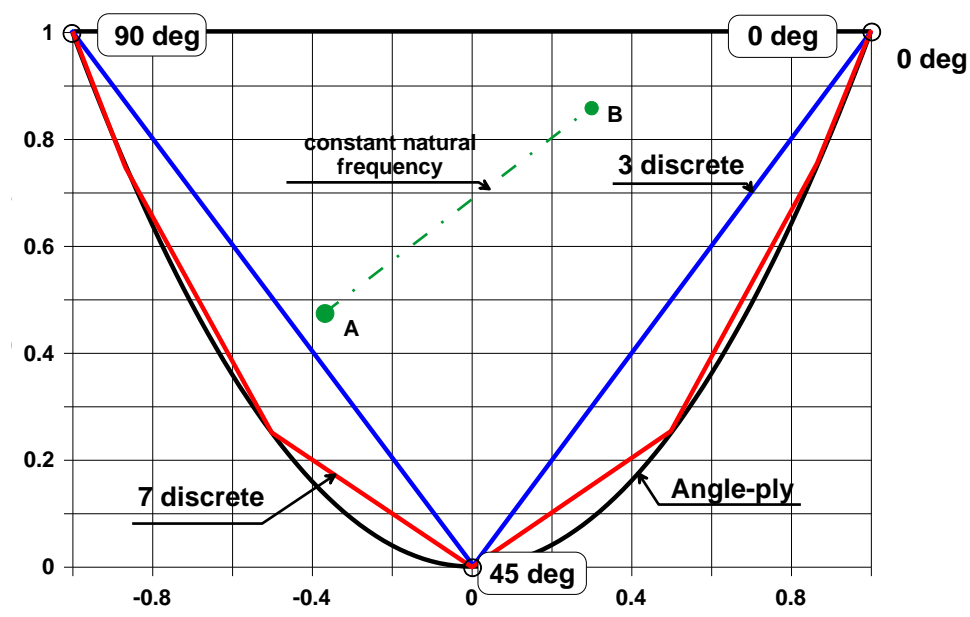

Figure 4. The design space for continuous and discrete design variables.

For three discrete design variables, using the definition in Equation $(7 \mathrm{c})$ and the previous assumptions, the stiffnesses $D_{16}$ and $D_{26}$ can be written in the following way:

$$
D_{16}=D_{26}=\frac{1}{2} \frac{1}{3}\left(\frac{t}{N}\right)^{3} U_{2}\left[-\sum_{p=2,4,6}^{N / 2} 6(p-1)+\sum_{p=N / 2+2, N / 2+4,}^{N} 6(p-1)\right] .
$$

Let us note that the total number of design variables is reduced to three, i.e., $x, y$, and $D_{16}$. However, the value $D_{16}$ is not arbitrary and can be derived by algebraic manipulations with the aid of the following identity:

$$
\sum_{k=1}^{N}[3 k(k-1)+1]=N^{3} y+N^{3} \sum_{p}[3 p(p-1)+1]\left(\text { plies oriented at } \pm 45^{\circ}\right)=N^{3}
$$


To demonstrate it, let us consider the laminate $[0 / 0 /+45 /-45 / 0 / 0 / 90 / 90 / 90 / 90 / 0 / 0 /-45 /+45 /$ $0 / 0](N=16)$. From Equation $(18): 4096 x=(1+7+61+91+331+397+631+721)\left(0^{\circ}\right)-(127+169+$ $217+271)\left(90^{\circ}\right)=2240-784=1456 ; 4096 y=2240+784=3024$. It is necessary to find the locations of plies oriented at $\pm 45^{\circ}$, the sum of which is equal to $4096-3024=1072$. The method of this procedure is described by Muc $[48,49]$ and in the Appendix A. For the known location of plies oriented at $\pm 45^{\circ}$, one can derive the values of $D_{16}$. Finally, the Rayleigh-Ritz quotient (Equation (13)) is a function of two design variables- $x$ and $y$.

Since the Rayleigh-Ritz quotient (Equation (13)) is a linear function of the two design variables $x$ and $y$, two points A and B having identical values of $\omega^{2}$ can be joined only by a straight line in the Euclidean space. Thus, for three discrete variables, the contour plots constitute a set of straight parallel lines (see Figure 4). It is possible to extend those lines to the whole interior of the parabola. For angle-ply laminates, the maximum/minimum of the natural frequency corresponds to the global maximum/minimum of the symmetric laminated plates, although it is not unique.

\subsection{Optimal Stacking Sequences}

As a numerical example, the material properties of graphite/epoxy composites are used: $E_{1}=98.0 \mathrm{GPa} ; E_{2}=7.90 \mathrm{GPa} ; G_{12}=5.60 \mathrm{GPa} ; v_{12}=0.28 ; \rho=1520 \mathrm{~kg} / \mathrm{m}^{3}$.

For simply supported plates (SSSS), the resulting fundamental frequencies (Equation (13)) are of the closed form

$$
\omega_{m n}^{2}=\frac{\pi^{4}}{\rho}\left[D_{11}\left(\frac{m}{L_{x}}\right)^{4}+2\left(D_{12}+2 D_{66}\right)\left(\frac{m n}{L_{x} L_{y}}\right)^{2}+D_{22}\left(\frac{n}{L_{y}}\right)^{4}\right]
$$

assuming that the terms $A_{16}=A_{26}=B_{16}=B_{26}=0$.

Figure 5 shows the distributions of the first natural frequency with the ply orientation for angle-ply symmetric stacking sequences-the first eigenmode $(m=n=1)$. The maximal fundamental frequency varies with the geometrical ratio $L_{x} / L_{y}$. The maximum occurs at $\theta=0^{\circ}$ for $L_{x} / L_{y}<0.7$. The increase of the $L_{x} / L_{y}$ ratio results in the increase of the maximal fiber orientations to the value $\theta=90^{\circ}$ as $L_{x} / L_{y}=1.4$. For the prescribed value of the $L_{x} / L_{y}$ ratio, the maximum of the frequency lies on the parabola $y=x^{2}$ and the straight line tangent to the parabola (Figure 6). The explicit form of the family of straight lines can be found from Equation (21) by inserting Equation (19). The slope of the contour lines depends on the position of the maximum (see Figure 6).

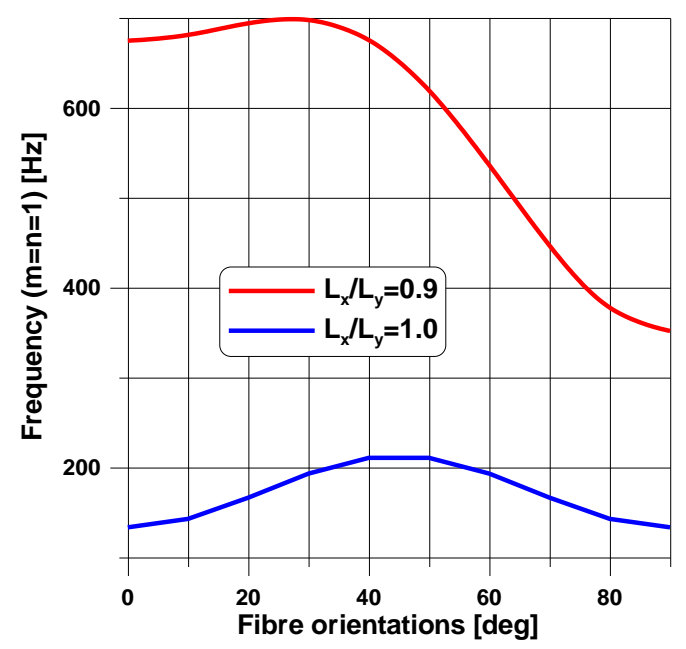

Figure 5. Distributions of eigenfrequencies for angle-ply simply supported (SSSS) plates.

The analytical results plotted in Figures 5 and 6 are compared with the results of the finite element (FE) analysis carried out with the use of nine-noded quadrilateral FEs denoted as the NKTP 32 (the FE 
program NISA II v.17 [50])). The finite element NKTP 32 includes transverse shear effects of the first order $\left(G_{13}=G_{23}=0.5 G_{12}\right)$. For $N=12$, the difference between numerical and analytical computations does not exceed 2-3\%. In addition, let us note that in the numerical analysis, the $D_{16}$ and $D_{26}$ terms are not equal to zero. The green triangle demonstrates the boundaries of three discrete design planes.

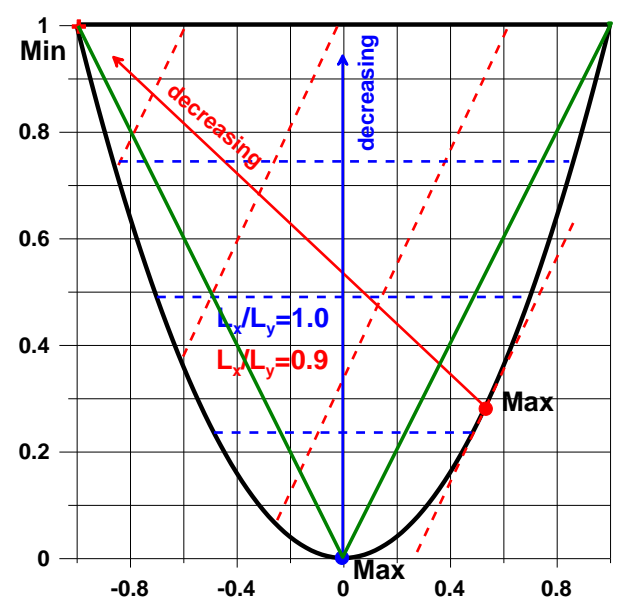

Figure 6. Contours of natural frequencies on the $x, y$ plane for angle-ply and symmetric laminates-simply supported (SSSS) plates.

The change of the boundary conditions from SSSS to angle-ply clamped (CCCC) leads to the change of the positions of the maxima and the minima of natural frequencies (see Figures 7 and 8). The maximum always occurs for fibers oriented at $\theta=0^{\circ}$ or $\theta=90^{\circ}$. The minimal eigenfrequency is inside the interval $(0,90)$ and its position depends on the value of the $L_{x} / L_{y}$ ratio. As the $L_{x} / L_{y}$ ratio increases, the position of the minimum is shifted from $\theta=90^{\circ}$ to $\theta=0^{\circ}$, and $\theta=45^{\circ}$ for $L_{x} / L_{y}=1$.

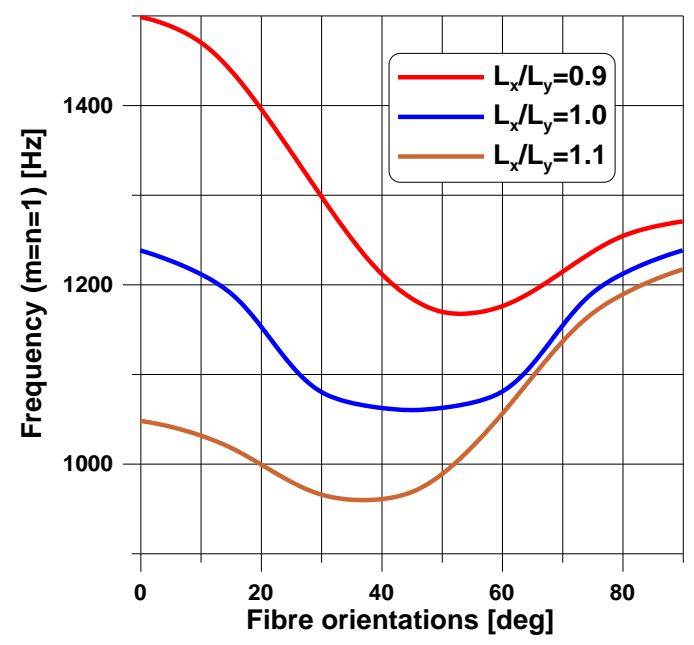

Figure 7. Distributions of eigenfrequencies for angle-ply clamped (CCCC) plates.

The numerical results confirm the previous theoretical considerations, i.e., the contour plots are represented by straight lines and the maximum/minimum eigenfrequencies lie at the boundary of the parabola (the angle-ply configurations).

The next problem deals with the analysis of free-clamped (FFFC) rectangular plates (see Figures 9 and 10) that refer directly to the analysis of aeroelastic problems for composite wings applied to aircraft main structures. The numerical analysis demonstrates that the global minimum/maximum exists only independently of the plate aspect ratio. The maximal eigenfrequency occurs for plates having fibers oriented at $\theta=90^{\circ}$ and the minimal at $\theta=0^{\circ}$. 


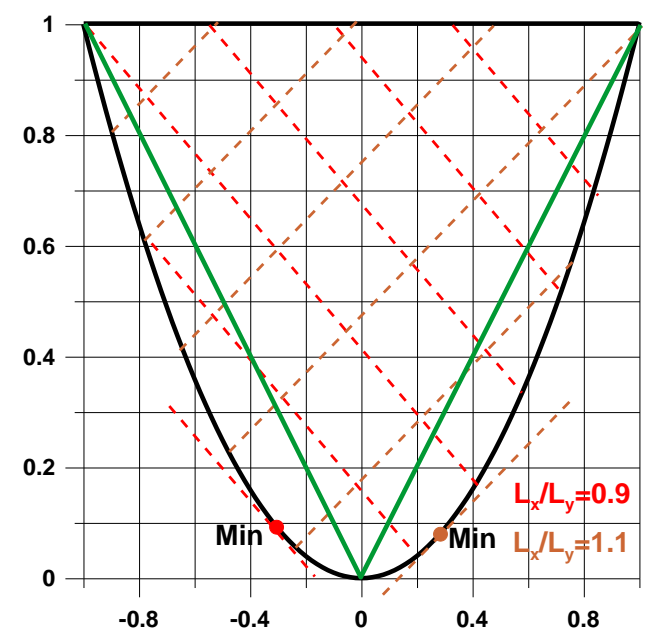

Figure 8. Contours of natural frequencies on the $x, y$ plane for angle-ply and symmetric laminates-clamped (CCCC) plates.

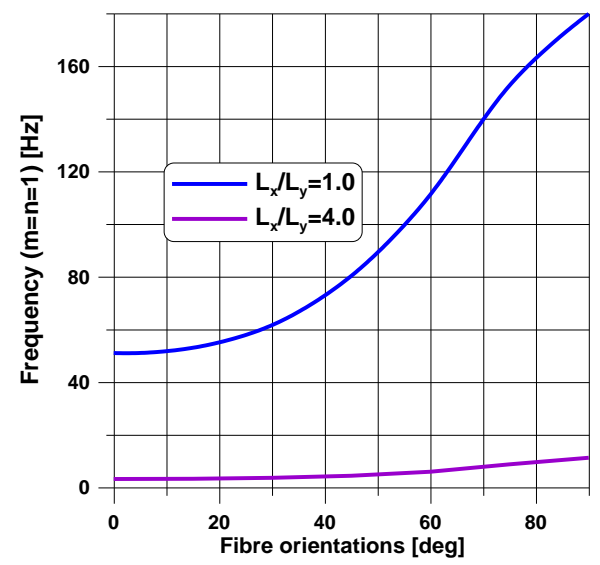

Figure 9. Distributions of eigenfrequencies for angle-ply free-clamped (FFFC) plates.

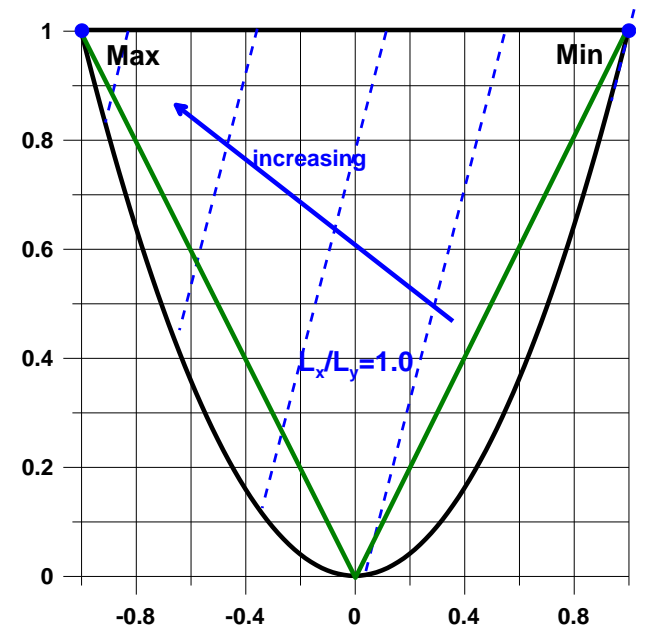

Figure 10. Contours of natural frequencies on the $x, y$ plane for and symmetric laminates-free-clamped (FFFC) plates.

Again, similarly as previously, the contour plots are represented by straight lines and the maximum/ minimum eigenfrequencies lie at the boundary of the parabola (the angle-ply configurations). 


\subsection{Variable Stiffness}

In this section, the analysis of the influence of composite patches for optimal eigenfrequencies is carried out, and the simulation is implemented for an FFFC plate. Mechanical characteristics of the system are identical to those detailed previously. The plate is made of the host composite plate with area $A_{h}$ and thickness $t_{h}$. It is reinforced by rectangular composite patches covering area $A_{r}$ (symmetric with respect to the host mid-plane) and having thickness $t_{r}$. The laminate is assumed to be angle-ply so that fiber orientations are characterized by one variable representing fiber orientation $\theta$. The results of the numerical FE analysis are plotted in Figure 11.

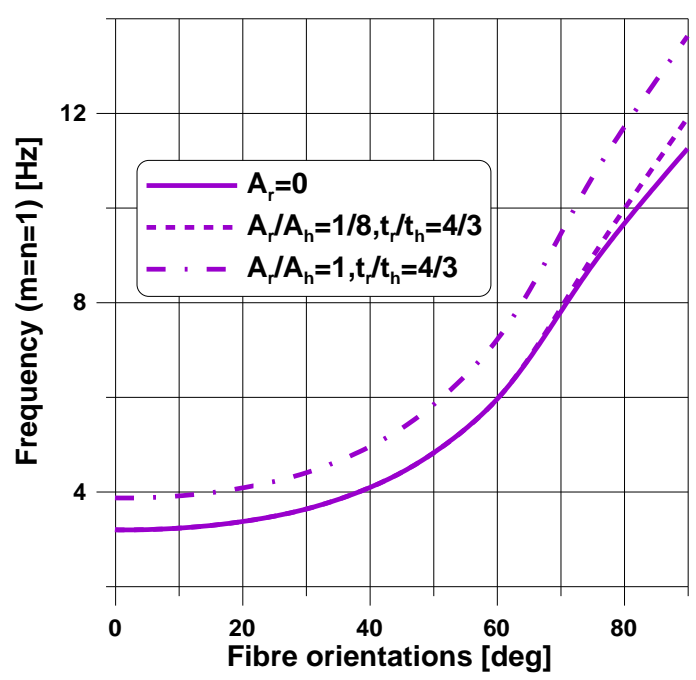

Figure 11. The effects of patch reinforcement on the distributions of eigenfrequencies-angle-ply plate (FFFC, $\left.L_{x} / L_{y}=4\right)$.

The growth of the thickness on the whole area of the plate results in increase of the eigenfrequencies (see Equation (13)). The reinforcement with the use of the local rectangular patches leads to local increases of the natural frequencies. It is possible to optimize the location of the local patches, their shape and positions, as well as their stacking sequences-detailed analysis is presented in Refs. [27-32].

The analysis of the locally reinforced plates was conducted with the use of 3D solid FE. Figure 12 demonstrates eigenmodes obtained for the host and reinforced plates. The analysis is conducted for plates oriented at $\theta=0^{\circ}$-it corresponds to the longer axis of the plate. The orientation is identical for unreinforced and reinforced plate. The displacements equal to zero are located at the clamped edge and are denoted by the dark blue color. As may be observed, the change of the mode shape is not significant-almost identical positions of the minimal and maximal (the red color) displacements. For the host plate the eigenmodes are presented on the left side of the picture. 


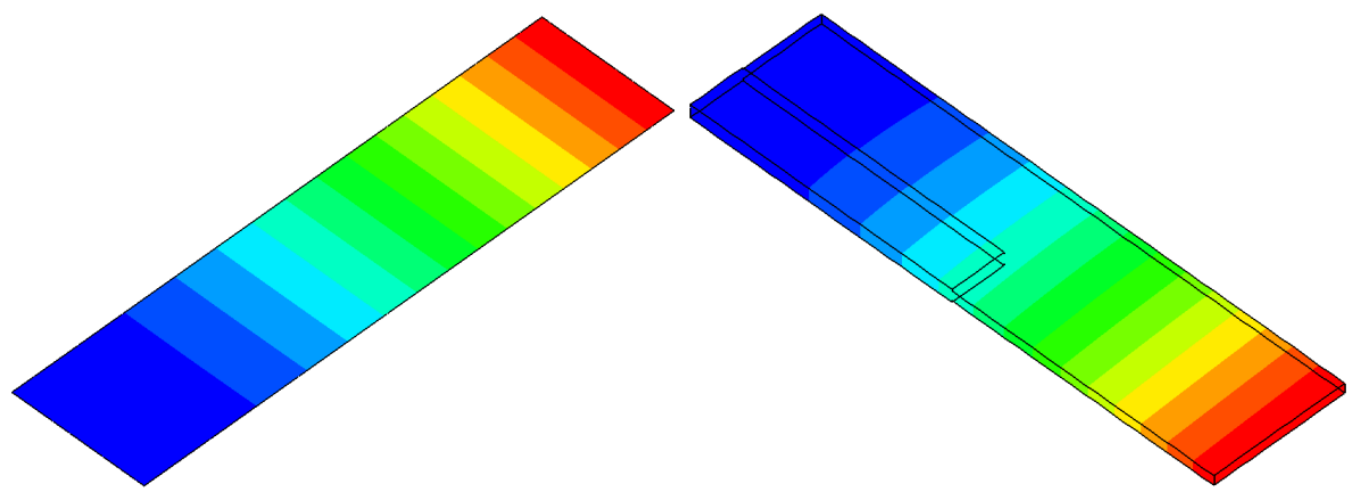

(a) Mode I

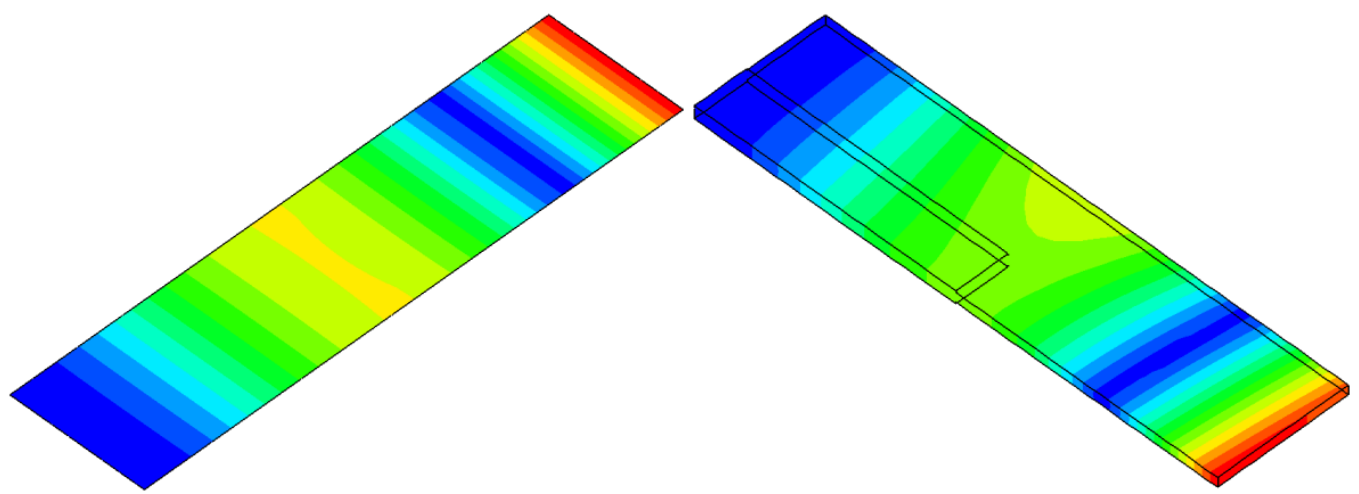

(b) Mode II

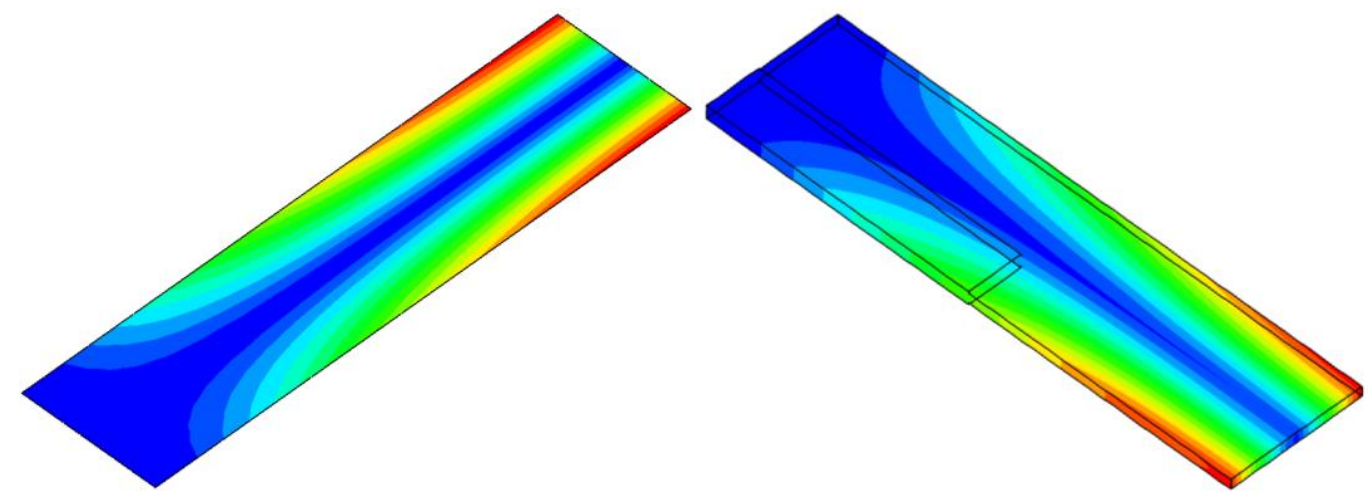

(c) Mode III

Figure 12. Mode shapes of the unreinforced and reinforced plates $\left(\theta=0, L_{x} / L_{y}=4\right.$, FFFC plate).

\section{Conclusions}

This paper proposes an efficient stacking sequence optimization tool for angle-ply and symmetrically laminated plates. The strategy is based on the introduction of two new design variables, and a two-step continuous or discrete optimization. The feasible region of allowable solutions has the shape of a parabola for the continuous design variables or a triangle for three discrete design variables.

For the angle-ply fiber orientations, the maximal/minimal values of the natural vibrations occur at the parabola independently from the prescribed boundary conditions (clamped, simply supported, or free-clamped (FFFC)), but the location of those values is a function of the aspect ratio $L_{x} / L_{y}$ and of the material properties. In the design space, the curves characterizing the constant eigenfrequencies have the form of straight lines. 
It is demonstrated that for discrete variables, the number of lamination parameters (otherwise four) is reduced to two only.

The above conclusions are valid for the constant-stiffness problems, i.e., plates made of straight fibers and having constant thickness on the whole structure, called the host plate. It is demonstrated that the increase of the eigenfrequencies can be achieved by variable stiffness using additional local composite strips and tapered construction of the plate, or by adding piezoelectric layers to control by voltage the structural response.

The advantage of the proposed approach over the classical lamination parameters depends on the use of the design parameters attributed directly to the ply thicknesses and orientations.

For composite laminated 2D structures the variety of problems connected with the definitions of appropriate design variables, objectives functions and optimization algorithms is discussed in Refs [51-55].

Funding: This research was funded by the Ministry of Higher Education (Poland) grant number M-03/ $331 / 2018 /$ DS.

Conflicts of Interest: The authors declare no conflict of interest.

\section{Appendix A}

Construction of the Required Eigenfrequencies and Stacking Sequences

Let us assume that the plate geometry, material properties, and boundary conditions are prescribed in advance by the designer. The plate is made of $N$ discrete plies $\left(0_{2}, \pm 45,90_{2}\right)$ constituting symmetric laminates and having identical thicknesses $(t / N)$ and material properties. The procedure can be conducted in the following way:

1. Choose point A belonging to the edges of the triangle (Figure A1);

2. Find the appropriate stacking sequence corresponding to the value $\mathrm{x}_{\mathrm{A}}$; the fundamental two operations are given below (the package Mathematica):

$\mathrm{a}=$ Table $[3 * l *(l-1)+1,\{l, N / 4\}]$;

$\mathbf{f}=$ Subsets $[\mathbf{a},\{\mathbf{L}\}]$

First of all, a list of values of the expression " $3 \times l(l-1)+1$ " is generated when the natural number $l$ runs from 1 to $N / 4$; then, a finite number of subsets " $\mathrm{f}$ " having exactly " $\mathrm{L}$ " elements are constructed from the list " $a$ " - a numerical example is given below;

3. Compute the value of the eigenfrequency corresponding to the assumed stacking sequence-the chosen subset f;

4. At the design space $(x, y)$, find the point $\mathrm{B}$ belonging to the parabola (angle-ply fiber orientations) which has the identical value of the eigenfrequency as that for symmetric laminates corresponding to the subset $\mathrm{f}$ and computed at the previous step;

5. Join the points A and B (Figure A1);

6. Draw a set of straight lines parallel to the straight line AB;

7. Choose the appropriate value of the eigenfrequency (the parameter of the parallel lines) and the point $C$;

8. Find the stacking sequence corresponding to the point $\mathrm{C}$. 


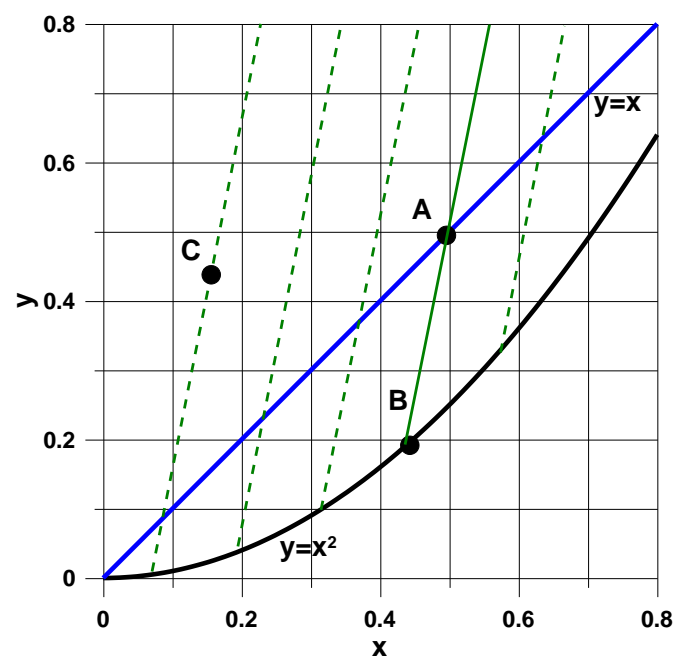

Figure A1. Construction of the required eigenfrequencies and stacking sequences.

Numerical Example

Let us choose the point $\mathrm{A}$ that has the coordinates $y_{\mathrm{A}}=x_{\mathrm{A}}=0.4793(\mathrm{~N} / 4=16)$. Thus, $x_{\mathrm{A}}{ }^{*}(N / 4)^{3}=1963.21$. There are 36 subsets " $\mathrm{f}$ " that represent the value 1963. One of them can be written in the following way $\{91,127,169,217,331,397,631\}$ and for $N / 4=16$, it corresponds to the stacking sequence $\{\div, \div, \div, \div, \div, 91,127,169,217, \div, 331,397, \div, \div, 631, \div\}-$ one of the possible forms of the table "a". In the table "a", the nonzero terms are replaced by the pairs oriented at $0^{\circ}$, whereas the symbol " $\div$ " by the pairs oriented at $\pm 45^{\circ}$. For the above symmetric laminate configuration, one can compute the value of the eigenfrequency. The identical value of the eigenfrequency can be obtained for the 35 other laminate configurations having identical values of the $D_{11}, D_{12}, D_{22}$ and $D_{66}$ terms in the bending stiffness matrix. By the FE computations it can be easily verified that the nonzero terms $D_{16}$ and $D_{26}$ have negligible effects on the values of the eigenfrequencies for all 36 laminate configurations. For the angle-ply configuration, the point $B$ has an identical eigenfrequency value to that of the symmetric laminate configuration defined above. Then, it is possible to plot the set of parallel lines parametrized by the value of the eigenfrequency (see Figure A1). For any chosen point (let us say C) it is possible to derive the discrete laminate configuration. Let us note that from the definition of the parameters $x$ and $y$ (Equation (17)), the parameters corresponding to the orientations $0^{\circ}$ and $90^{\circ}$ are derived from the relations $x_{C}\left(0^{\circ}\right)=0.5^{*}\left(x_{C}+y_{C}\right)^{*}(N / 4)^{3}, x_{C}\left(90^{\circ}\right)=0.5^{*}\left(y_{C}-x_{C}\right)^{*}(N / 4)^{3}$. In addition, the sum of the discrete plies $n\left(0^{\circ}\right)+n\left(90^{\circ}\right)+n\left(45^{\circ}\right)$ is equal to $N / 4$. It is necessary to remember that the solution, understood in the sense of the stacking sequence, is not unique.

\section{References}

1. Lovejoy, A.E. Natural Frequencies and an Atlas of Mode Shapes for Generally Laminated, Thick, Skew, Trapezoidal Plates. Master's Thesis, Virginia Polytechnic Institute and State University, Blacksburg, VA, USA, 1994.

2. Fantuzzi, N.; Tornabene, F.; Bacciocchi, M.; Ferreira, A.J.M. On the Convergence of Laminated Composite Plates of Arbitrary Shape through Finite Element Models. J. Compos. Sci. 2018, 2, 16. [CrossRef]

3. Yu, Y.-Y. Vibrations of Elastic Plates: Linear and Nonlinear Dynamical Modeling of Sandwiches, Laminated Composites, and Piezoelectric Layers; Springer: New York, NY, USA, 1996.

4. Kapania, R.K.; Raciti, S. Recent advances in analysis of laminated beams and plates. Part II: Vibrations and wave propagation. AIAA J. 1989, 27, 935-945. [CrossRef]

5. Reddy, J.N.; Khdeir, A.A. Buckling and vibration of laminated composite plates using various plate theories. AIAA J. 1989, 27, 1809-1817. [CrossRef] 
6. Tornabene, F.; Fantuzzi, N.; Bacciocchi, M.; Viola, E. Higher-order theories for the free vibrations of doubly-curved laminated panels with curvilinear reinforcing fibers by means of a local version of the GDQ method. Compos. Part B Eng. 2015, 81, 196-230. [CrossRef]

7. Tornabene, F.; Fantuzzi, N.; Bacciocchi, M. Foam core composite sandwich plates and shells with variable stiffness: Effect of the curvilinear fiber path on the modal response. J. Sandw. Struct. Mater. 2017. [CrossRef]

8. Grandhi, R. Structural optimization with frequency constraints-A review. AIAA J. 1993, 31, $2296-2303$. [CrossRef]

9. Szyszkowski, W.; King, J.M. Optimization of frequencies spectrum in vibrations of flexible structures. AIAA J. 1993, 31, 2163-2168.

10. Bert, C.W. Optimal design of a composite material plate to maximize its fundamental frequency. J. Sound Vib. 1977, 50, 229-237. [CrossRef]

11. Bert, C.W. Design of clamped composite-material plates to maximize fundamental frequency. J. Mech. Des. 1978, 100, 274-278. [CrossRef]

12. Reiss, R.; Ramachandran, S. Maximum frequency design of symmetric angle-ply laminates. Comput. Struct. 1987, 4, 476-487.

13. Grenestedt, J.L. Layup optimization and sensitivity analysis of the fundamental eigenfrequency of composite plates. Compos. Struct. 1989, 12, 193-209. [CrossRef]

14. Wright, J.R.; Cooper, J.E. Introduction to Aircraft Aeroelasticity and Loads; John Wiley \& Sons: Chichester, UK, 2007.

15. Chowdary, T.V.; Sinha, P.K.; Parthan, S. Finite element flutter analysis of composite skew panels. Comput. Struct. 1996, 58, 613-620. [CrossRef]

16. Forster, E.E.; Yang, H.T. Flutter control of wing boxes using piezoelectric actuators. J. Aircraft 1998, 35, 949-957. [CrossRef]

17. Moosavi, M.R.; Oskouei, A.N.; Khelil, A. Flutter of subsonic wing. Thin Walled Struct. 2005, 43, 617-627. [CrossRef]

18. Zhao, H.; Cao, D. A study on the aero-elastic flutter of stiffened laminated composite panel in the supersonic flow. J. Sound Vib. 2013, 332, 4668-4679. [CrossRef]

19. Alyanak, E.J.; Pendleton, E. A design study employing aeroelastic tailoring and an active aeroelastic wing design approach on a tailless lambda wing configuration. In Proceedings of the 15th AIAA/ISSMO Multidisciplinary Analysis and Optimization Conference, Atlanta, GA, USA, 16-20 June 2014.

20. Singha, M.K.; Ganapathi, M. A parametric study on supersonic flutter behavior of laminated composite skew flat panels. Compos. Struct. 2005, 69, 55-63. [CrossRef]

21. Hyer, M.W.; Charette, R.F. Use of curvilinear fiber format in composite structure design. In Proceedings of the 30th Structures, Structural Dynamics, and Material Conference, Mobile, AL, USA, 3-5 April 1989; pp. 1011-1015.

22. Abdalla, M.M.; Setoodeh, S.; Gurdal, Z. Design of variable stiffness composite panels for maximum fundamental frequency using lamination parameters. Compos. Struct. 2007, 81, 283-291. [CrossRef]

23. Kameyama, M.; Fukunaga, H. Optimum design of composite plate wings for aeroelastic characteristics using lamination parameters. Compos. Struct. 2007, 85, 213-224. [CrossRef]

24. Chandra, R.; Chopra, I. Structural modeling of composite beams with induced-strain actuators. AIAA J. 1993, 31, 1692-1701. [CrossRef]

25. Brunelle, E.J.; Robertson, S.R. Initially stressed mindlin plates. AIAA J. 1974, 12, 1036-1045. [CrossRef]

26. Yang, I.H.; Shieh, J.A. Vibrations of initially stressed thick rectangular orthotropic plates. J. Sound Vib. 1987, 119, 545-558. [CrossRef]

27. Rammerstorfer, F.G. Increase of the first natural frequency and buckling load of plates by optimal fields of initial stresses. Acta Mech. 1977, 27, 217-238. [CrossRef]

28. Shah, P.H.; Ray, M.C. Active control of laminated composite truncated conical shells using vertically and obliquely reinforced 1-3 piezoelectric composites. Eur. J. Mech. A Solids 2012, 32, 1-12. [CrossRef]

29. Kędziora, P.; Muc, A. Optimal shapes of PZT actuators for laminated structures subjected to displacement or eigenfrequency constraints. Compos. Struct. 2012, 94, 1224-1235. [CrossRef]

30. Muc, A.; Kędziora, P. Optimal Design of Smart Laminated Composite Structures. Mater. Manuf. Process. 2010, 25, 272-280. [CrossRef] 
31. Muc, A.; Kędziora, P.; Stawiarski, A. Buckling enhancement of laminated composite structures partially covered by piezoelectric actuators. Eur. J. Mech. A Solids 2019, 73, 112-125. [CrossRef]

32. Mota Soares, C.M.; Mota Soares, C.A.; Franco Correia, V.M. Optimal design of piezolaminated structures. Compos. Struct. 1999, 47, 625-634. [CrossRef]

33. Ganesan, R.; Zabihollah, A. Vibration analysis of tapered composite beams using a higher-order finite element: I. Formulation. Compos. Struct. 2007, 77, 306-318. [CrossRef]

34. Ganesan, R.; Zabihollah, A. Vibration analysis of tapered composite beams using a higher-order finite element: II. Parametric study. Compos. Struct. 2007, 77, 319-330. [CrossRef]

35. Kapania, R.K.; Singhvi, S. Free vibration analyses of generally laminated tapered skew plates. Compos. Eng. 1992, 2, 197-212. [CrossRef]

36. Viglietti, A.; Zappino, E.; Carrera, E. Free vibration analysis of locally damaged aerospace tapered composite structures using component-wise models. Compos. Struct. 2018, 192, 38-51. [CrossRef]

37. Muc, A. Design of blended/tapered multilayered structures subjected to buckling constraints. Compos. Struct. 2018, 186, 256-266. [CrossRef]

38. Babu, A.A.; Vasudevan, R. Vibration analysis of rotating delaminated non-uniform composite plates. Aerosp. Sci. Technol. 2017, 60, 172-182. [CrossRef]

39. Muc, A. Optimal design of composite multilayered plated and shell structures. Thin-Walled Struct. 2007, 45, 816-820. [CrossRef]

40. Whitney, J.M.; Leissa, A.W. Analysis of heterogeneous anisotropic plates. ASME J. Appl. Mech. 1969, 36, 261-266. [CrossRef]

41. Grenestedt, J.L. Layup Optimization of Composite Structures; Report No. 92-24; Royal Institute of Technology: Stockholm, Sweden, 1992.

42. Li, J.; Narita, Y. Analysis and optimal design for supersonic composite laminated plate. Compos. Struct. 2013, 101, 35-46. [CrossRef]

43. Miki, M.; Sugiyama, Y. Optimum design of laminated composite plates using lamination parameters. AIAA J. 1993, 31, 921-922. [CrossRef]

44. Fukunaga, H.; Vanderplaats, G.N. Stiffness Optimization of Orthotropic Laminated Composites Using Lamination Parameters. AIAA J. 1991, 29, 641-648. [CrossRef]

45. Diaconu, C.G.; Sekine, H. Layup optimization for buckling of laminated composite shells with restricted layer angles. AIAA J. 2004, 42, 2153-2158. [CrossRef]

46. Diaconu, C.G.; Sato, M.; Sekine, H. Buckling characteristics and layup optimization of long laminated composite cylindrical shells subjected to combined loads using lamination parameters. Compos. Struct. 2002, 58, 423-433. [CrossRef]

47. Bloomfield, M.W.; Diaconu, C.G.; Weaver, P.M. On feasible regions of lamination parameters for lay-up optimization of laminated composites. Proc. R. Soc. A Math. Phys. Eng. Sci. 2009, 465, 1123-1143. [CrossRef]

48. Muc, A. Choice of design variables in the stacking sequence optimization for laminated structures. Mech. Compos. Mater. 2016, 52, 211-224. [CrossRef]

49. Muc, A.; Chwał, M. Analytical discrete stacking sequence optimization of rectangular plates subjected to buckling and FPF constraints. J. Theor. Appl. Mech. 2016, 54, 423-436. [CrossRef]

50. NISA II User's Manual; Engineering Mechanics Research Corporation: Troy, MI, USA, 1993.

51. Muc, A.; Ulatowska, A. Local fibre reinforcement of holes in composite multilayered plates. Compos. Struct. 2012, 94, 1413-1419. [CrossRef]

52. Muc, A.; Muc-Wierzgoń, M. An evolution strategy in structural optimization problems for plates and shells. Compos. Struct. 2012, 94, 1461-1470. [CrossRef]

53. Muc, A.; Muc-Wierzgoń, M. Discrete optimization of composite structures under fatigue constraints. Compos. Struct. 2015, 133, 834-839. [CrossRef]

54. Muc, A. Evolutionary design of engineering constructions. Latin Am. J. Solids Struct. 2018, 15, 87. [CrossRef]

55. Muc, A. Peculiarities in the material design of buckling resistance for tensioned laminated composite panels with elliptical cut-outs. Materials 2018, 11, 1019. [CrossRef] [PubMed]

(C) 2018 by the author. Licensee MDPI, Basel, Switzerland. This article is an open access article distributed under the terms and conditions of the Creative Commons Attribution (CC BY) license (http:/ / creativecommons.org/licenses/by/4.0/). 